\title{
Extensor mechanism's anatomy at the metacarpophalangeal joint
}

\begin{abstract}
The digital extensor mechanism at the level of the MCP joint consists of the extensor tendon, sagittal bands, and volar plate. The sagittal bands are part of a complex extensor retinacular system that includes the triangular ligament between the lateral bands, the transverse retinacular ligament, and the oblique retinacular ligament at the proximal interphalangeal (PIP) joint level.
\end{abstract}

Keywords: Extensor Mechanism; Metacarpophalangeal Joint
Volume 8 Issue 4 - 2017

\section{Konstantinos Ditsios, Panagiotis \\ Konstantinou, losafat Pinto, Argyrios Karavelis, Lazaros Kostretzis}

Department of Orthopaedics, General Hospital G Papanikolaou, Aristotle University of Thessaloniki, Greece

\begin{abstract}
Correspondence: Konstantinos Ditsios, Assistant Professor, First Orthopaedic Department, Aristotle University of Thessaloniki, G. Papanikolaou General Hospital, Exohi, GR 57010 Thessaloniki, Greece, Email ditsiosk@otenet.gr
\end{abstract}

Received: May 22, 2017| Published: July 05, 2017
Abbreviations: MCP: Metacarpophlangeal, PIP: Proximal Interphalangeal, EDC: Extensor Digitorum Communis, EIP: Extensor Indicis Proprius, EDM: Extensor Digiti Minimi

\section{Introduction}

Safe surgical dissection and exposure require an in-depth knowledge of anatomy. In no place is this more relevant than in the surgical approaches to the hand. The anatomy of the hand can be discussed in many ways and in extensive detail. For the discussion in this article anatomy will focus on the metacarpophalangeal joint

\section{Discussion}

The finger extensor system has nine zones; zone 5 overlies the MCP joints.

Proximally to the MCP joint level, stout interconnecting bands exist between the ring EDC to the small and middle fingers, and there is a less substantial band from the middle to the index finger. These juncturaetendinae restrict independent extension of the ring and middle fingers when the other digits are flexed at the MCP joints and also preserve finger extension when the EDC is lacerated proximal to them.

The metacarpophalangeal (MCP) joint is a multiaxial diarthrodialcondyloid joint. The metacarpal head is asymmetric with the radial condyle being larger, allowing ulnar slope of the head. Additionally, the metacarpal head is longer and broader at its volar surface, producing the cam phenomenon, that tights the collateral ligaments when the joint is flexed. The capsule of the MCP joint extends from the metacarpal neck to the base of the proximal phalanx producing volar and larger dorsal synovial reflections. Volarly the MCP Joint is supported by the volar plate. Collateral and intervolar plate ligaments reinforce from the lateral side the volar plate and produce a linked ligament support between adjacent MCP joints.

The sagittal bands arise from the volar plate of the MP joint, the intermetacarpal ligaments and the volar periosteum, to insert on the extensor hood. They are $15-20 \mathrm{~mm}$ long, become film at the level of the MC head enveloping the extensor tendons and maintaining their central position. The ulnar sagittal bands are stronger and denser. There are oblique and transverse fibers in the sagittal bands. The oblique fibers transit volarly and distally to insert on the lateral side of the proximal phalanx, collateral ligament and volar plate. These oblique fibers allow proximal and distal migration of the whole extensor mechanism, facilitating MCP motion.

The typical arrangement of finger extensor tendons is as follows: (1) single EIP inserting ulnar to the EDC tendon of the index finger; (2) single tendon from the EDC to the index and middle fingers; (3) double tendon from the EDC to the ring finger; (4) absent EDC to small finger, but usually a substantial juncturatendinum from the ring EDC to EDM; and (5) double EDM tendon to the little finger with a double insertion into the MCP joint hood. ${ }^{2}$ Distal to MCP joint the EDC tendons are trifurcated into a central slip and in two lateral slips. The central slip travels distally to insert onto the central tubercle of the middle phalanx. There is not a direct insertion of the tendon on the base of the proximal phalanx, but only loose connective tissue. ${ }^{3}$ Extension of the MP joint is transmitted by the pull of the EDC tendon through the sagittal bands. When the sagittal band system is damaged the EDC tendon subluxates or dislocates off and MP joint extension is compromised.

The intrinsic tendons (lumbricals and interossei) form along each lateral surface of the finger the lateral bands. Their course is along the proximal phalanx from volar to dorsal. At the MCP joint level the intrinsic muscles and tendons are palmar to the joint axis of rotation. This bimodal course allows the intrinsics to flex the MCP joints and extend the IP. The lateral bands naturally slide dorsally and volarly with finger flexion and extension respectively and are held in position at the PIP level by the transverse retinacular ligament. Proximal to the PIP these lateral bands join up the lateral slips of the EDC tendon. ${ }^{4}$ These conjoined lateral bands coalesce over the middle phalanx and continue distally to become the terminal tendon.

\section{Functional anatomy}

Extension of the fingers at the MP joint is exclusively a function of the extrinsic extensor tendons. Extension of the PIP joints, although primarily a function of the intrinsic muscles, can also occur through the extrinsic tendon extensor system, provided that MP joint hyperextension is blocked. When intrinsic palsy allows the MP joint to hyperextend, the extrinsic extensor system becomes insufficient to extend the IP joints, and a "claw finger" results. Extension at the MP joint is possible regardless of the position of flexion of the PIP and DIP joints. 
Low ulnar paralysis results in weakness of thumb pinch, little and ring finger claw deformity (Duchenne sign), loss of the normal pattern of finger flexion, and significant loss of hand dexterity and strength. In high ulnar paralysis loss of active ring and little DIP joint flexion and wrist flexion and dorsal loss of sensation compound the aforementioned findings, although paradoxically, the claw deformity tends to be less severe. However, in a low medial nerve palsy patient's index and middle finger cannot flex at the MCP joint. This is known as hand of benediction or median claw hand. Also, the thenar muscles become paralyzed and are subsequently flattened. While the adductor pollicis remains intact the thumb becomes adducted and laterally rotated (ape-hand deformity) (Figure $1 \& 2$ ).

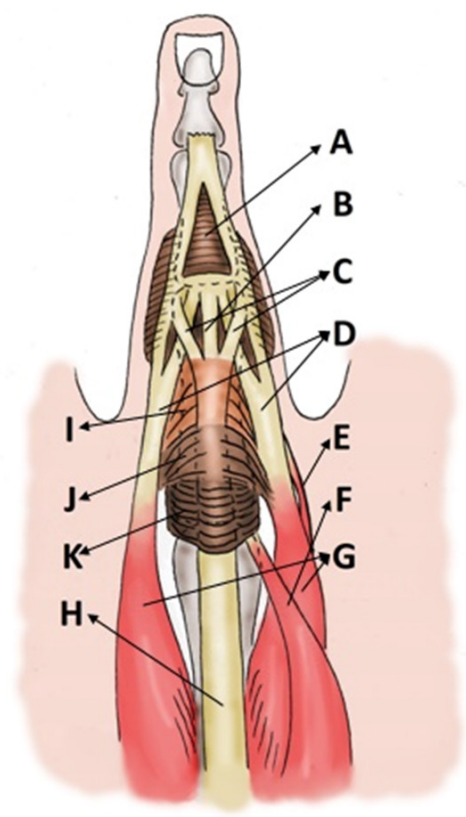

Figure I Extensor Mechanism, dorsal view.

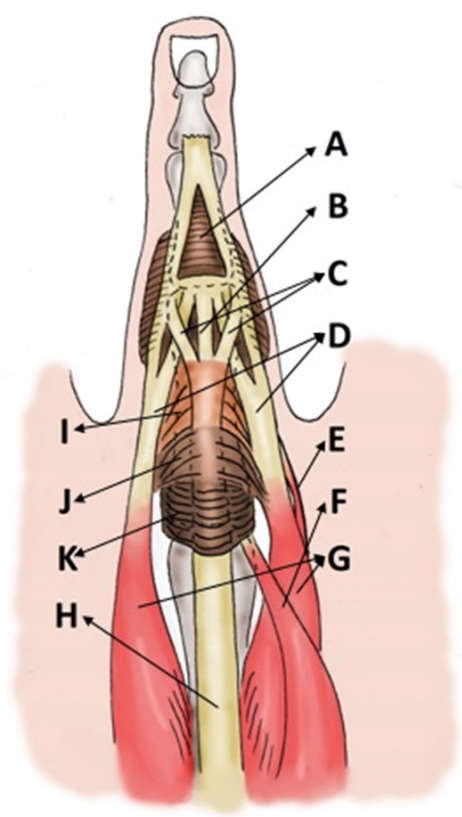

a: triangular ligament; b: central slip; c: slips of long extensor tendon to lateral bands; d: lateral bands; e: lumbrical muscle; f: superficial belly of dorsal interosseous muscle; g: deep belly of dorsal interosseous muscle or palmar interosseous muscle; h: long extensor tendon; i: oblique fibers; j: transverse fibers; k: saggital bands; l: oblique retinacular ligament (Landmeer's ligament)

\section{Conclusion}

Traumatic injury to the extensor mechanism of the hand can sometimes be an insoluble problem. Unique to the hand is the complex relationship of not only the muscles and tendons overlying bone but also close proximity and delicate balance of accessory anatomic structures including vessels and nerves. Therefore deep comprehension of regional anatomy is of crucial importance.

\section{Conflicts of interest and acknowledgement}

The authors declare that they have no conflict of interest.

\section{Acknowledgments}

None.

\section{Conflicts of interest}

None.

\section{References}

1. Hirai Y, Yoshida K, Yamanaka K, et al. An anatomic study of the extensor tendons of the human hand. J Hand Surg. 2001;26(6):1009-1015.

2. Von Schroeder HP, Botte MJ. Anatomy of the extensor tendons of the fingers: variations and multiplicity. J Hand Surg. 1995;20(1):27-34.

3. Van Sint Jan S, Rooze M, Van Audekerke J, et al. The insertion of the extensor digitorum tendon on the proximal phalanx. J Hand Surg. 1996;21(1):69-76.

4. Schultz RJ, Furlong 2nd J, Storace A. Detailed anatomy of the extensor mechanism at the proximal aspect of the finger. $J$ Hand Surg. $1981 ; 6(5): 493-498$

\footnotetext{
Figure 2 Extensor mechanism, lateral view.
} 